\title{
Clinical characteristics and survival of patients concurrently diagnosed with lung cancer and active pulmonary tuberculosis
}

Hwa Young Lee

Catholic University of Korea School of Medicine https://orcid.org/0000-0002-1582-2256

Jung Won Heo

Catholic University of Korea

Hye Seon Kang

catholic university of korea

Ji Young Kang

Catholic university of Korea

Jin Woo Kim

Catholic University of Korea

Sang Haak Lee

Catholic University of Korea

Seung Joon Kim

Catholic University of Korea

Chang Dong Yeo ( $\sim$ brainyeo@catholic.ac.kr)

Catholic University of Korea https://orcid.org/0000-0002-4103-7921

Research article

Keywords: Lung cancer, Mycobacterium tuberculosis, Neoplasm staging

Posted Date: March 2nd, 2021

DOI: https://doi.org/10.21203/rs.3.rs-279237/v1

License: (a) This work is licensed under a Creative Commons Attribution 4.0 International License.

Read Full License 


\section{Abstract \\ Background}

Active pulmonary tuberculosis (TB) occasionally coexists with lung cancer. However, the clinical and radiologic characteristics of this cooccurrence have not been fully evaluated.

\section{Methods}

Patients diagnosed with lung cancer and active pulmonary TB from January 2009 to December 2017 in four hospitals of the Catholic University of Korea were retrospectively reviewed. The clinical characteristics, including the TB diagnosis methods, lung cancer pathology, staging, initial radiographic features, and survival were analyzed and compared to 575 lung cancer patients without active pulmonary TB from the same hospitals.

\section{Results}

Forty-eight (0.48\%) of the 9,936 lung cancer patients had active pulmonary TB confirmed by positive culture results or polymerase chain reaction analysis for $M$. tuberculosis at the time of the initial cancer diagnosis. The majority of the patients (95.9\%) had non-small cell lung cancer and $56.2 \%$ of the lung cancers were located in the both upper lobes. In the initial computed tomography, the most frequent findings were a mass-like lesion (79.2\%) and separate nodules (75\%). When compared to lung cancer patients without TB, the body mass index (BMI) was lower (21.4 vs. 23.1, $P=0.001$ ) in patients with TB. Moreover, the lung cancer patients with TB had advanced clinical stages compared to patients without TB; T3-4 (70.9\% vs. 50.6\%, $P=0.002)$, N2-3 (85.2\% vs. 55.6\%, P<0.001); M1 (65.9\% vs. $44.5 \%, P=0.007)$. Patients' age, histology, location, and the presence of epidermal growth factor receptor mutations were not statistically different between the two groups. Interestingly, lung cancer with TB was associated with lower mortality (hazard ratio $=0.35,95 \% \mathrm{Cl}: 0.21-0.60$ ).

\section{Conclusions}

Rarely diagnosed concurrent active tuberculosis in lung cancer patients was associated with lower BMI and advanced clinical stages. Active investigation of and treatment for active pulmonary TB in lung cancer patients could improve patient outcomes.

\section{Background}

Globally, lung cancer is a leading cause of death, and active pulmonary tuberculosis (TB) is an important comorbidity in lung cancer patients. Especially in South Korea, the annual incidence of TB was 
46/100,000 people in 2019 (1), which was considered an intermediate incidence country. Therefore, early diagnosis and treatment of pulmonary TB are important.

The risk of pulmonary TB in patients with lung cancer was reported to be $5-6 \%$ (2) or 83/100,000 people (incidence rate ratio $=9$ ) in a recent meta-analysis (3). Adults with old pulmonary tuberculosis had an elevated risk of lung cancer (hazard ratio $(\mathrm{HR})=3.24,95 \% \mathrm{Cl}$ : $1.87-5.62$ ) compared to the general population without TB in a Korean National Cancer Incidence Database study (4). Interestingly, patients with old TB lesions had a higher incidence of epidermal growth factor receptor (EGFR) mutations in adenocarcinoma than those without $(P=0.018)(5)$. However, the impact of coexisting pulmonary TB on the survival of lung cancer patients was controversial in previous studies. Based on a report published in Turkey, there was no change in survival in 38 of 3,350 TB patients with concomitant lung cancer (6). A paper from Taiwan showed that active TB was an independent predictor of better survival with an HR of 0.68 (95\% Cl: 0.48-0.97) (7). A study by Jian et al. based on a Chinese database reported that male lung adenocarcinoma patients with coexisting pulmonary diseases including TB had an elevated risk of mortality (8).

The diagnosis of pulmonary TB in patients suspected of lung cancer is difficult because of the similarities in signs, symptoms, and radiologic results, which may delay appropriate treatment (9-11). In one report, the average delay in the diagnosis of lung cancer in patients with TB was 6-9 months (12). To shorten this delay, it is important to know the differences in clinical and radiologic characteristics between the patients with and without pulmonary TB when making a lung cancer diagnosis. We previously experienced a case of TB reactivation suspected to be cancer progression during oral tyrosine kinase inhibitor treatment in a patient diagnosed with non-small cell lung cancer (NSCLC) (13). Concurrent pulmonary TB in lung cancer patients results in delays in scheduled chemotherapy, radiation, or surgical intervention. However, there is a lack of clinical and radiologic evidence to differentiate active pulmonary TB in patients during the lung cancer diagnosis process. To identify a way to rule out active pulmonary TB in lung cancer patients, we performed a retrospective chart review of patients diagnosed with lung cancer at four university hospitals. The clinical characteristics including the TB diagnosis methods, lung cancer pathology, staging, and initial radiographic features were analyzed and compared to those of lung cancer patients without active pulmonary TB from the same hospitals.

\section{Methods}

\section{Data collection}

Patients concurrently diagnosed with active TB were identified based on the International Codes of Disease 10th Edition Clinical Modification (ICD-10-CM). We identified patients diagnosed with lung cancer in four hospitals (Seoul St. Marys' Hospital, Eunpyeong St. Mary's Hospital, Uijeongbu St. Marys' Hospital, and Bucheon St. Mary's Hospital) of the Catholic University of Korea using ICD code C34, and pulmonary TB using ICD codes A15 and A16 from January 2009 to December 2017. A retrospective chart review was performed to select the patients diagnosed with active pulmonary TB within three months of the initial 
work-up for lung cancer prior to the start of cancer treatment. The diagnosis of active pulmonary TB was determined by the results of acid-fast bacillus (AFB) culture, analysis of TB in sputum by polymerase chain reaction (PCR) or bronchoscopic wash fluid samples, and pathology review of a percutaneous needle biopsy or surgical specimen. Among 9,936 lung cancer patients, 61 were concurrently diagnosed with pulmonary TB at the time of the initial lung cancer diagnosis, and 48 had positive TB cultures or TB PCR results. Methods on TB diagnosis, lung cancer pathology including mutation status, stages, and initial radiologic features of the chest computed tomography (CT) were collected. The radiology findings of primary lung cancer were reviewed based on the CT results of radiology specialists, by two experienced pulmonologists (HY Lee and CD Yeo) and decisions were reached by consensus. The location, size, and characteristics of the tumor, the presence of calcification, cavitation, separate nodules, pleural effusion, and mediastinal lymphadenopathy were assessed retrospectively. Metastatic lymphadenopathy was defined as the short diameter of a lymph node $\geq 1 \mathrm{~cm}$. Parenchymal lesions that were calcified or demonstrated fibrotic changes were considered old TB sequelae.

\section{Registry data}

To analyze the differences between the 48 lung cancer patients who had active pulmonary TB and the patients without TB, we used the data from 575 lung cancer patients collected at the same hospital during the same period. This study used the Catholic Medical Center (CMC) lung cancer registry containing data from seven hospitals (Seoul St. Marys' Hospital, Yeouido St. Mary's Hospital, Eunpyeong St. Mary's Hospital, Uijeongbu St. Marys' Hospital, Bucheon St. Mary's Hospital, Incheon St. Mary's Hospital, and St. Vincent's Hospital) in the capital region of South Korea. The CMC lung cancer registry has been consecutively enrolling patients with lung cancer confirmed by tissue biopsy since October 2014 and is currently ongoing (14-16). We analyzed data of the lung cancer patients without TB diagnosed at Seoul, Eunpyeong, Bucheon, and Uijeongbu from January 2009 to December 2017. The data included in the present analysis were obtained in August 2019. At the initial lung cancer diagnosis, the demographic and clinical data including age, sex, smoking history, underlying diseases, and history of pulmonary tuberculosis were collected. Clinical staging was performed using the 7th edition of the TNM staging system, which was authorized by the American Joint Committee on Cancer (17). This study was approved by the Clinical Research Ethics Committee of the Catholic Medical Center (approval number: XC20RIDI0057P).

\section{Statistical analysis}

For continuous variables, the mean and standard deviation were calculated for normally distributed variables, whereas the median and interquartile range (IQR: 25th - 75th ) were used for non-normally distributed data. Proportions were used for categorical variables. Analysis of the differences between the lung cancer patients with and without pulmonary TB was performed using the Student's $t$-test for continuous variables and the $\chi^{2}$ test or Fisher's exact test for categorical variables. Odds ratios (ORs) and their $95 \%$ confidence intervals (Cls) were computed. All tests were two-sided, and a $P$-value $<0.05$ was taken to indicate statistical significance. All statistical analyses were performed using the SPSS for Windows software package (ver. 24.0; SPSS, Inc., Chicago, IL, USA). 


\section{Results}

Table 1 shows the baseline clinical characteristics of the 48 patients who had active pulmonary TB at the time of the initial lung cancer diagnosis. The mean age was $71.3 \pm 10.1$ years, and $39(81.3 \%)$ patients were male with a mean body mass index (BMI) of $21.4 \pm 2.9$. There were $11(25 \%)$ current smokers and 19 (39.6\%) ex-smokers. Twelve (25\%) patients had diabetes mellitus (DM) and eight (16.7\%) had a history of previous TB treatment. Three (6.3\%) patients had combined silicosis in the lung and one (2.1\%) was gastrectomy status. The majority of the patients (73\%) were diagnosed with active pulmonary TB based on positive AFB cultures of the sputum or bronchoscopic wash fluid. Eleven patients had positive TB PCR results in bronchoscopic wash fluid or sputum and two patients had both positive TB PCR results and granulomatous inflammation by pathology review in lung tissues. All these 13 patients were treated with anti-TB medication. The most common pathologic types of lung cancer with concurrent active pulmonary TB were squamous cell carcinoma (50\%) and adenocarcinoma (43.8\%). Although $45.8 \%$ of the patients had not had EGFR mutation tests, about half of the tested patients (41.7\%) did not have EGFR mutations in their lung cancer tissue. During the $361.9 \pm 431.8$ days of follow up, $32(66.7 \%)$ were survived. 
Table 1

Baseline clinical characteristics of total 48 patients concurrently diagnosed lung cancer and active pulmonary tuberculosis

\begin{tabular}{|l|l|}
\hline & Mean \pm SD or median (IQR) \\
\hline No (\%)
\end{tabular}

HIV: human immunodeficiency virus

TB-PCR: tuberculosis-polymerase change reaction

EGFR: epidermal growth factor receptor 


\begin{tabular}{|ll|}
\hline & Mean \pm SD or median (IQR) \\
& No (\%) \\
\hline Lung cancer cell, $\mathrm{n}(\%)$ & $21(43.8)$ \\
Adenocarcinoma & $24(50)$ \\
Squamous cell carcinoma & $2(4.2)$ \\
Small cell carcinoma & $1(2.1)$ \\
Adenosquamous carcinoma & $0(0)$ \\
\hline Large cell carcinoma & \\
\hline EGFR mutation status & $6(12.5)$ \\
Positive & $20(41.7)$ \\
Negative & $22(45.8)$ \\
\hline Not done & \\
\hline Total follow up duration (days) & $186(66-493)$ \\
\hline Survived, $n$ (\%) & $32(66.7)$ \\
\hline HIV: human immunodeficiency virus & \\
\hline TB-PCR: tuberculosis-polymerase change reaction \\
\hline EGFR: epidermal growth factor receptor \\
\hline
\end{tabular}

Table 2 summarizes the initial staging and radiologic findings of the 48 patients in which active TB was identified during the lung cancer diagnosis process. Based on the 7th edition of lung cancer TNM staging system (17), $29(60 \%)$ of the patients were stage IV and $14(29.2 \%)$ were stage III. Only $10 \%$ were stage I or II. The clinical lymph node stages also showed that 25 (52.1\%) patients had N3 and $16(35.3 \%)$ had N2. The most frequent tumor location was the left upper lobe (LUL). The initial CT findings showed that $19(39.6 \%)$ patients had old TB lesions and $12(25 \%)$ had pleural effusion. The most common finding of lung cancer was a mass $(79.2 \%)$, separate nodules $(75.0 \%)$, consolidation $(27.1 \%)$, and cavitation (14.6\%). Only two (4.2\%) showed ground-glass opacity. 
Table 2

Initial staging and radiologic findings of the lung cancer patients concurrently diagnosed lung cancer and active pulmonary tuberculosis

\begin{tabular}{|c|c|}
\hline & No (\%) \\
\hline Lung cancer initial stage, n (\%) & $4(8.3)$ \\
\hline I & $1(2.1)$ \\
\hline II & $14(29.2)$ \\
\hline III & $29(60.4)$ \\
\hline \multicolumn{2}{|l|}{ IV } \\
\hline Clinical lymph node stage, n (\%) & $3(8.3)$ \\
\hline NO & $4(8.3)$ \\
\hline N1 & $16(33.3)$ \\
\hline N2 & $25(52.1)$ \\
\hline \multicolumn{2}{|l|}{ N3 } \\
\hline Lung cancer location, n (\%) & $11(22.9)$ \\
\hline RUL & $4(8.3)$ \\
\hline RML & $11(22.9)$ \\
\hline RLL & $16(33.3)$ \\
\hline LUL & $6(12.5)$ \\
\hline \multicolumn{2}{|l|}{ LLL } \\
\hline Lung cancer radiologic finding, $\mathrm{n}(\%)$ & $7(14.6)$ \\
\hline Cavity & $38(79.2)$ \\
\hline Mass & $13(27.1)$ \\
\hline Consolidation & $2(4.2)$ \\
\hline Ground glass opacity & $36(75.0)$ \\
\hline Separate nodule & $12(25.0)$ \\
\hline Pleural effusion & $19(39.6)$ \\
\hline Old tuberculosis & \\
\hline
\end{tabular}

RUL: right upper lobe, RML: right middle lobe, RLL: right lower lobe, LUL: left upper lobe, LLL: left lower lobe. 
We compared the demographic and lung cancer characteristics between the patients with active pulmonary TB and those without in Table 3. Age, sex, and previous pulmonary TB history were not statistically different between the two groups. Interestingly, BMI was significantly lower in the TB group $(21.4 \pm 2.9$ vs. $23.1 \pm 3.2, P=0.001)$. In the patients diagnosed with lung cancer, cell type, tumor location, and EGFR mutation status were not statistically different. However, in the initial clinical staging, the pulmonary TB group had more stage III or IV patients $(P=0.03)$ and also more T3-4 $(\mathrm{OR}=2.38,95 \% \mathrm{Cl}$ : 1.2-4.5), N2-3 (OR $=5.46,95 \% \mathrm{Cl}: 2.3-13.1)$ and $\mathrm{M} 1$ disease $(\mathrm{OR}=2.41,95 \% \mathrm{Cl}: 1.3-4.6)$ than the nonTB patients. During the follow-up periods (TB vs. non-TB: median 186 days vs. 421 days), the percentage of patients who died was lower in the TB group than in the non-TB (TB vs. non-TB: 16 (33.3\%) vs. 324 $(56.4 \%), P<0.002)$. In Kaplan-Meier survival analysis, there was no statistical significance in overall survival when stratified by pulmonary TB diagnosis (Fig. 1). However, the multivariate Cox proportional hazards model revealed that the presence of TB at the initial diagnosis of lung cancer was associated with lower mortality ( $\mathrm{HR}=0.35,95 \% \mathrm{Cl}: 0.21-0.60)$ than without TB. In addition, male sex $(\mathrm{HR}=2.41,95 \%$ $\mathrm{Cl}: 1.77-3.27)$, old age $(\mathrm{HR}=1.06,95 \% \mathrm{Cl}: 1.05-1.08)$, lower $\mathrm{BMI}(\mathrm{HR}=0.93,95 \% \mathrm{Cl} 0.90-0.97), \mathrm{T} 2(\mathrm{HR}=$ $2.03,95 \% \mathrm{Cl} 1.33-3.09), \mathrm{T} 3(\mathrm{HR}=2.15,95 \% \mathrm{Cl}: 1.38-3.35), \mathrm{T} 4(\mathrm{HR}=2.69,95 \% \mathrm{Cl}: 1.75-4.10), \mathrm{N} 2(\mathrm{HR}=$ $2.01,95 \% \mathrm{Cl}: 1.42-2.86), \mathrm{N} 3(\mathrm{HR}=2.96,95 \% \mathrm{Cl}: 2.04-4.05)$, and $\mathrm{M} 1$ ( $\mathrm{HR}=2.58,95 \% \mathrm{Cl}: 1.99-3.35)$ stages were independently associated with all-cause mortality in the lung cancer patients (Fig. 2). 
Table 3

Comparison of the lung cancer patients between concurrent diagnosed pulmonary tuberculosis (TB) or not

\begin{tabular}{|c|c|c|c|c|c|}
\hline & $\begin{array}{l}\text { Pulmonary TB } \\
(\mathrm{n}=48)\end{array}$ & $\begin{array}{l}\text { Non-TB } \\
(n=575)\end{array}$ & $\begin{array}{l}\text { Odds } \\
\text { ratio }\end{array}$ & $95 \% \mathrm{Cl}$ & $P$ \\
\hline Age (yr) & $71.3 \pm 10.1$ & $68.1 \pm 10.1$ & & & 0.06 \\
\hline Male, n (\%) & $39(81.3)$ & $438(76.2)$ & 1.36 & $0.6-2.9$ & 0.48 \\
\hline Body mass index $\left(\mathrm{kg} / \mathrm{m}^{2}\right)$ & $21.4 \pm 2.9$ & $23.1 \pm 3.2$ & & & 0.001 \\
\hline Previous tuberculosis history & $8(16.7)$ & $86(19.4)$ & 0.83 & $0.4-1.8$ & 0.85 \\
\hline Lung cancer cell, n (\%) & $21(43.8)$ & $280(48.7)$ & & & 0.07 \\
\hline Adenocarcinoma & $24(50.0)$ & $189(32.9)$ & & & \\
\hline Squamous cell carcinoma & $2(4.2)$ & 79 (13.7) & & & \\
\hline Small cell carcinoma & $1(2.1)$ & $4(0.7)$ & & & \\
\hline Adenosquamous carcinoma & $0(0)$ & $6(1.0)$ & & & \\
\hline \multicolumn{6}{|l|}{ Large cell carcinoma } \\
\hline Lung cancer location, n (\%) & $11(22.9)$ & $134(25.6)$ & & & 0.60 \\
\hline RUL & $4(8.3)$ & $37(7.1)$ & & & \\
\hline RML & $11(22.9)$ & $141(26.9)$ & & & \\
\hline RLL & $16(33.3)$ & $123(23.5)$ & & & \\
\hline LUL & $6(12.5)$ & $89(17.0)$ & & & \\
\hline \multicolumn{6}{|l|}{ LLL } \\
\hline EGFR mutation status, n (\%) & $2(7.7)$ & $62(14.4)$ & 0.50 & $0.1-2.2$ & 0.56 \\
\hline 19 deletion & $1(3.8)$ & $45(10.4)$ & 0.34 & $0.0-2.6$ & 0.50 \\
\hline L858R & & & & & \\
\hline
\end{tabular}

RUL: right upper lobe, RML: right middle lobe, RLL: right lower lobe, LUL: left upper lobe, LLL: left lower lobe.

EGFR: epidermal growth factor receptor

${ }^{\dagger}$ T3-4 versus T1-2

${ }^{\ddagger} \mathrm{N} 2-3$ versus $\mathrm{N} 1-2$

`M1 versus $\mathrm{M} 0$ 


\begin{tabular}{|c|c|c|c|c|c|}
\hline & $\begin{array}{l}\text { Pulmonary TB } \\
(n=48)\end{array}$ & $\begin{array}{l}\text { Non-TB } \\
(n=575)\end{array}$ & $\begin{array}{l}\text { Odds } \\
\text { ratio }\end{array}$ & $95 \% \mathrm{Cl}$ & $P$ \\
\hline Lung cancer initial stage, $\mathrm{n}(\%)$ & $4(8.3)$ & $132(23.0)$ & & & 0.03 \\
\hline I & $1(2.1)$ & $46(8.0)$ & & & \\
\hline II & $14(29.2)$ & $140(24.4)$ & & & \\
\hline III & $29(60.4)$ & $256(44.6)$ & & & \\
\hline \multicolumn{6}{|l|}{ IV } \\
\hline Lung cancer TNM stage, n (\%) & $34(70.8)$ & $288(50.6)$ & 2.38 & $1.2-4.5$ & $0.01^{\dagger}$ \\
\hline T3-4 & $14(29.2)$ & $282(49.5)$ & 5.46 & $2.3-13.1$ & \multirow{3}{*}{$\begin{array}{l}0.01^{\ddagger} \\
0.01^{q}\end{array}$} \\
\hline T1-2 & $41(85.4)$ & $318(55.6)$ & 2.41 & $1.3-4.6$ & \\
\hline N2-3 & $6(12.8)$ & $254(44.4)$ & & & \\
\hline N0-1 & $29(65.9)$ & $256(44.5)$ & & & \\
\hline M1 & $15(34.1)$ & $319(55.5)$ & & & \\
\hline \multicolumn{6}{|l|}{ MO } \\
\hline All-cause mortality, n (\%) & $16(33.3)$ & $324(56.4)$ & 0.39 & $0.2-0.7$ & 0.002 \\
\hline Follow up duration (days) & $186(66-493)$ & $421(206-734)$ & & & $<0.001$ \\
\hline \multicolumn{6}{|c|}{$\begin{array}{l}\text { RUL: right upper lobe, RML: right middle lobe, RLL: right lower lobe, LUL: left upper lobe, LLL: left lowe } \\
\text { lobe. }\end{array}$} \\
\hline \multicolumn{6}{|c|}{ EGFR: epidermal growth factor receptor } \\
\hline \multicolumn{6}{|l|}{${ }^{\dagger} \mathrm{T} 3-4$ versus $\mathrm{T} 1-2$} \\
\hline \multicolumn{6}{|l|}{${ }^{\ddagger} \mathrm{N} 2-3$ versus N1-2 } \\
\hline ` $\mathrm{M} 1$ versus $\mathrm{M} 0$ & & & & & \\
\hline
\end{tabular}

\section{Discussion}

This study revealed that the patients with active pulmonary TB at the initial diagnosis of lung cancer had lower BMIs and more advanced clinical stages than those without pulmonary TB. Interestingly, tumor location and mutation status were not different between the groups. Moreover, a concurrent diagnosis of active TB was associated with lower all-cause mortality compared to the non-TB group in the Cox proportional hazards model.

In this study, a total of $48(0.48 \%)$ of the 9,936 lung cancer patients had active pulmonary TB at the initial diagnosis of lung cancer from 2009 to 2017 . The frequency of TB infection was approximately 4.8 per 
1,000 lung cancer diagnoses, which was lower than that in a study from Turkey $(1.1 \%$ of the total patients) (6). These incidences differed according to the burden of TB and underlying cancers. A study from Taiwan, a country with a high burden of TB, reported 6.8 per 1,000 person-years among the patients with hematologic malignancies after hematologic stem cell transplantation (18). The incidence was much lower in the United States, at 0.2 per 1,000 new cancer diagnoses (19). Based on these previous reports and our study results, the presence of active TB at the initial cancer diagnosis was relatively rare. However, the differential diagnosis of active TB is important in terms of diagnosis and cancer treatment.

Kmeid et al. reported that, from nine years of experience in a cancer center, $38 \%$ of 52 patients with active TB patients who had been referred to the institution due to suspicion of an underlying malignancy did not have cancer. Moreover, chemotherapy was delayed in $53 \%$ of the cancer patients who were to receive treatment and three patients died due to TB infection (19). Similarly, Kim et al. reported that coexisting pulmonary TB infections were located predominantly in the upper lobe, half of the lung cancer coexisting with pulmonary TB were at an advanced stage (IIIB or IV), and the average delay in diagnosing lung cancer was 11.7 (range, 1-24) months (11). In our study cohort, half of the patients with pulmonary TB had an upper lobe mass and much higher proportions of clinical stages T3-4, N2-3, and M1 than the nonTB group in the initial chest CT results. Moreover, since all 48 patients started anti-tuberculosis medication before or after a median of four days (IQR 1-19, data not shown) from the cancer diagnosis, the start of anti-cancer therapies was delayed in all cases.

The analysis of the characteristics of lung cancer in our study found that the TB patients were slightly older and had more squamous cell carcinoma than the non-TB patients without statistical significance. Although there were some previous reports that a past TB infection was associated with an elevated risk of lung cancer $(4,20-22)$, our study cohort showed that TB history had no association with a concurrent TB and lung cancer diagnosis in univariate analysis $(\mathrm{OR}=0.83,95 \% \mathrm{Cl}: 0.4-1.8)$. Differentiating pulmonary TB from a cancerous mass by chest CT is difficult and sometimes new cancer nodules can be masked by coexisting stable or active TB lesions (11). We tried to identify the differences in TB patients by location, pathology, and mutation status. However, none of the differences in these factors reached statistical significance.

The most interesting finding in our study was the all-cause mortality of the patients. Despite the advanced initial clinical staging of lung cancer patients with TB, the all-cause mortality was significantly lower than in patients without TB $(33.3 \%$ vs. $56.4 \%$; OR $=0.39,95 \% \mathrm{Cl}: 0.2-0.7)$. Since the follow-up duration was longer in the non-TB group, we performed a Kaplan-Meier survival analysis. The log-rank test revealed that the coexistence of pulmonary TB did not affect survival $(P=0.576)$. The Cox proportional hazards model showed a significant association of TB with all-cause mortality $(H R=0.35$, 95\% Cl: 0.21-0.60). However, the impact of TB on the prognosis of lung cancer patients may be controversial. Most population-based cohort studies reported that TB was an independent predictor of lung cancer death (adjusted $\mathrm{HR}=2.81,95 \% \mathrm{Cl}: 1.45-5.42$ for never smokers (23), $\mathrm{HR}=2.36 ; 95 \% \mathrm{Cl}$ : 1.13-4.91 (24), $\mathrm{HR}=1.06 ; 95 \% \mathrm{Cl}: 0.86-1.32$ (8)). One hospital-based study analyzed patients admitted to the TB department of the Chest Diseases and found no change in the overall survival of 38 of the 
3,350 patients newly diagnosed with lung cancer with concomitant active TB (6). A previous study that reviewed 276 culture-proven concomitant active TB and lung cancer patients found a longer median survival in NSCLC patients with active TB (HR=0.6,95\% Cl: 0.48-0.97) (7). These differences in the overall survival of lung cancer patients with or without TB could be because of the diagnosis of TB in the study cohorts. Population-based studies defined pulmonary TB by ICD codes without microbiological evidence. Moreover, patients infected with pulmonary TB due to immune suppression and/or poor health conditions during cancer treatment, which are associated with higher mortality would have been enrolled. Including our study, all of the three hospital-based studies enrolled patients diagnosed concurrently with active TB proved microscopically in the initial lung cancer diagnosis through a retrospective review. A population-based study cannot reflect real, microscopically proven active TB patients. Moreover, the patient populations differed in the timing of TB diagnosis and treatment.

It is also possible that the immunological effects of TB affect lung cancer. Kuo et al. found that active TB in squamous cell carcinoma increased the expression of CD3, CXCR3, and inducible protein-10, which are associated with $T$ cell recruitment, resulting in better survival outcomes of patients with lung cancer $(7$, 25-27). In our study, it was difficult to find an immunological factor associated with the better survival outcomes of lung cancer patients with TB. Therefore, further studies on this mechanism are needed. Otherwise, among our enrolled 48 patients with TB, the majority $(62.5 \%)$ of the patients received cancer treatment appropriate to the initial stages (27 received systemic chemotherapy and/or radiation therapy and three received curative resection). Moreover, all patients received immediate anti-TB medication with a median 4-day interval between anti-TB medication and lung cancer diagnosis. We assumed that the better overall survival of the lung cancer patients with TB in our study cohort must have been because of the active treatment of TB and lung cancer.

The strength of this study was that through an intensive chart review, we enrolled limited patients who had definite evidence of TB at the time of the initial diagnosis of lung cancer before starting cancer treatment. In addition, we used an ongoing multi-center lung cancer cohort database collected over nine years. These data represented patients from four university hospitals in the capital region of South Korea. Moreover, we compared the initial imaging as well as the clinical characteristics, pathologic findings, and EGFR mutation status between the patients with and without TB. As far as we know, our report was the first to use this patient population and study method. However, there were several limitations. First, the survival benefit shown in TB patients should not be generalized to culture-negative TB patients or patients diagnosed with TB during lung cancer treatment. Moreover, the survival analysis in this study was based on all-cause mortality. The mortality induced by active pulmonary TB should be analyzed separately to differentiate death due to TB infection from that due to lung cancer disease progression. Second, the combined analysis of the initial chest radiologic findings of the lung cancer patients without TB could have resulted in a more comprehensive understanding of the differences in lung cancer morphology between patients with and without TB.

\section{Conclusion}


Active TB found in the initial diagnosis of lung cancer patients was associated with lower BMI and advanced TNM stages compared to the non-TB group. However, the survival of lung cancer patients during treatment was better in the concurrently diagnosed TB patients. Active diagnosis and the immediate treatment of pulmonary TB during cancer staging work-up can lead to better survival outcomes in patients with lung cancer.

\section{Abbreviations}

TB

Tuberculosis

$\mathrm{BMI}$

Body mass index

HR

Hazard ratio

EGFR

Epidermal growth factor receptor

NSCLC

Non-small cell lung cancer

ICD

International codes of disease

AFB

Acid-fast bacillus

PCR

Polymerase chain reaction

CT

Computed tomography

CMC

Catholic Medical Center

ORs

Odds ratios

Cls

Confidence Intervals

DM

Diabetes mellitus

LUL

Left upper lobe

\section{Declarations}

Ethics approval and consent to participate 
Written informed consent for participation was obtained from all of the participants. This study was approved by the Clinical Research Ethics Committee of the Catholic Medical Center (approval number: XC20RIDI0057P).

\section{Consent for publication}

Not applicable.

\section{Availability of data and materials}

Data analyzed in the current study are not publicly available. They may be made available from the corresponding authors upon reasonable request.

\section{Competing interests}

The authors declare that they have no competing interests.

\section{Funding}

None

\section{Author contributions}

HY Lee performed statistical analysis and drafted the manuscript. JW Heo, HS Kang, JY Kang, JW Kim, SH Lee and SJ Kim collected patients' data and helped statistical analysis. CD Yeo designed and coordinated the study. All authors approved the final version of the manuscript.

\section{Acknowledgements}

Not applicable

\section{References}

1. Centers for disease control and prevention in Korea, 2019, Availabe at http://cdc.go.kr.

2. Ting YM, Church WR, Ravikrishnan KP. Lung carcinoma superimposed on pulmonary tuberculosis. Radiology. 1976;119(2):307-12.

3. Cheng MP, Abou Chakra CN, Yansouni CP, Cnossen S, Shrier I, Menzies D, et al. Risk of Active Tuberculosis in Patients with Cancer: A Systematic Review and Meta-Analysis. Clin Infect Dis. 2017;64(5):635-44.

4. Oh CM, Roh YH, Lim D, Kong HJ, Cho H, Hwangbo B, et al. Pulmonary Tuberculosis is Associated with Elevated Risk of Lung cancer in Korea: The Nationwide Cohort Study. J Cancer. 2020;11(7):1899-906. 
5. Luo YH, Wu CH, Wu WS, Huang CY, Su WJ, Tsai CM, et al. Association between tumor epidermal growth factor receptor mutation and pulmonary tuberculosis in patients with adenocarcinoma of the lungs. J Thorac Oncol. 2012;7(2):299-305.

6. Varol Y, Varol U, Unlu M, Kayaalp I, Ayranci A, Dereli MS, et al. Primary lung cancer coexisting with active pulmonary tuberculosis. Int J Tuberc Lung Dis. 2014;18(9):1121-5.

7. Kuo CH, Lo CY, Chung FT, Lee KY, Lin SM, Wang CH, et al. Concomitant active tuberculosis prolongs survival in non-small cell lung cancer: a study in a tuberculosis-endemic country. PLoS One. 2012;7(3):e33226.

8. Jian ZH, Huang JY, Ko PC, Jan SR, Nfor ON, Lung CC, et al. Impact of coexisting pulmonary diseases on survival of patients with lung adenocarcinoma: a STROBE-compliant article. Medicine (Baltimore). 2015;94(4):e443.

9. Zheng Z, Pan Y, Guo F, Wei H, Wu S, Pan T, et al. Multimodality FDG PET/CT appearance of pulmonary tuberculoma mimicking lung cancer and pathologic correlation in a tuberculosis-endemic country. South Med J. 2011;104(6):440-5.

10. Dasgupta P, Chakrabarti A, Halder D, Acharyya S, Gangopadhyay S. Results of diagnostic dilemma between lung cancer and sputum negative pulmonary tuberculosis: a retrospective study. J Indian Med Assoc. 2012;110(12):898-900.

11. Kim YI, Goo JM, Kim HY, Song JW, Im JG. Coexisting bronchogenic carcinoma and pulmonary tuberculosis in the same lobe: radiologic findings and clinical significance. Korean J Radiol. 2001;2(3):138-44.

12. Mok CK, Nandi P, Ong GB. Coexistent bronchogenic carcinoma and active pulmonary tuberculosis. J Thorac Cardiovasc Surg. 1978;76(4):469-72.

13. Lee HY, Kim JW, Yeo CD. A case of tuberculosis reactivation suspected of cancer progression during oral tyrosine kinase inhibitor treatment in a patient diagnosed as non-small cell lung cancer. $J$ Thorac Dis. 2017;9(8):E709-E13.

14. Heo JW, Kang HS, Park CK, Kim SK, Kim JS, Kim JW, et al. Regional emphysema score is associated with tumor location and poor prognosis in completely resected NSCLC patients. BMC Pulm Med. 2020;20(1):242.

15. Ban WH, Yeo CD, Han S, Kang HS, Park CK, Kim JS, et al. Impact of smoking amount on clinicopathological features and survival in non-small cell lung cancer. BMC Cancer. 2020;20(1):848.

16. Lim JU, Yeo CD, Kang HS, Park CK, Kim JS, Kim JW, et al. Prognostic value of platelet count and lymphocyte to monocyte ratio combination in stage IV non-small cell lung cancer with malignant pleural effusion. PLoS One. 2018;13(7):e0200341.

17. Edge SB, Compton CC. The American Joint Committee on Cancer: the 7th edition of the AJCC cancer staging manual and the future of TNM. Ann Surg Oncol. 2010;17(6):1471-4.

18. Fan WC, Liu CJ, Hong YC, Feng JY, Su WJ, Chien SH, et al. Long-term risk of tuberculosis in haematopoietic stem cell transplant recipients: a 10-year nationwide study. Int J Tuberc Lung Dis. 2015;19(1):58-64. 
19. Kmeid J, Kulkarni PA, Batista MV, El Chaer F, Prayag A, Ariza-Heredia EJ, et al. Active Mycobacterium tuberculosis infection at a comprehensive cancer center, 2006-2014. BMC Infect Dis. 2019;19(1):934.

20. Wu CY, Hu HY, Pu CY, Huang N, Shen HC, Li CP, et al. Pulmonary tuberculosis increases the risk of lung cancer: a population-based cohort study. Cancer. 2011;117(3):618-24.

21. Yu YH, Liao CC, Hsu WH, Chen HJ, Liao WC, Muo CH, et al. Increased lung cancer risk among patients with pulmonary tuberculosis: a population cohort study. J Thorac Oncol. 2011;6(1):32-7.

22. Hong S, Mok Y, Jeon C, Jee SH, Samet JM. Tuberculosis, smoking and risk for lung cancer incidence and mortality. Int J Cancer. 2016;139(11):2447-55.

23. Leung CC, Hui L, Lee RS, Lam TH, Yew WW, Hui DS, et al. Tuberculosis is associated with increased lung cancer mortality. Int J Tuberc Lung Dis. 2013;17(5):687-92.

24. Heuvers ME, Aerts JG, Hegmans JP, Veltman JD, Uitterlinden AG, Ruiter R, et al. History of tuberculosis as an independent prognostic factor for lung cancer survival. Lung Cancer. 2012;76(3):452-6.

25. Ma J, Liu L, Che G, Yu N, Dai F, You Z. The M1 form of tumor-associated macrophages in non-small cell lung cancer is positively associated with survival time. BMC Cancer. 2010;10:112.

26. Whiteside TL. Immune responses to malignancies. J Allergy Clin Immunol. 2010;125(2 Suppl 2):S272-83.

27. Walrath J, Zukowski L, Krywiak A, Silver RF. Resident Th1-like effector memory cells in pulmonary recall responses to Mycobacterium tuberculosis. Am J Respir Cell Mol Biol. 2005;33(1):48-55.

\section{Figures}




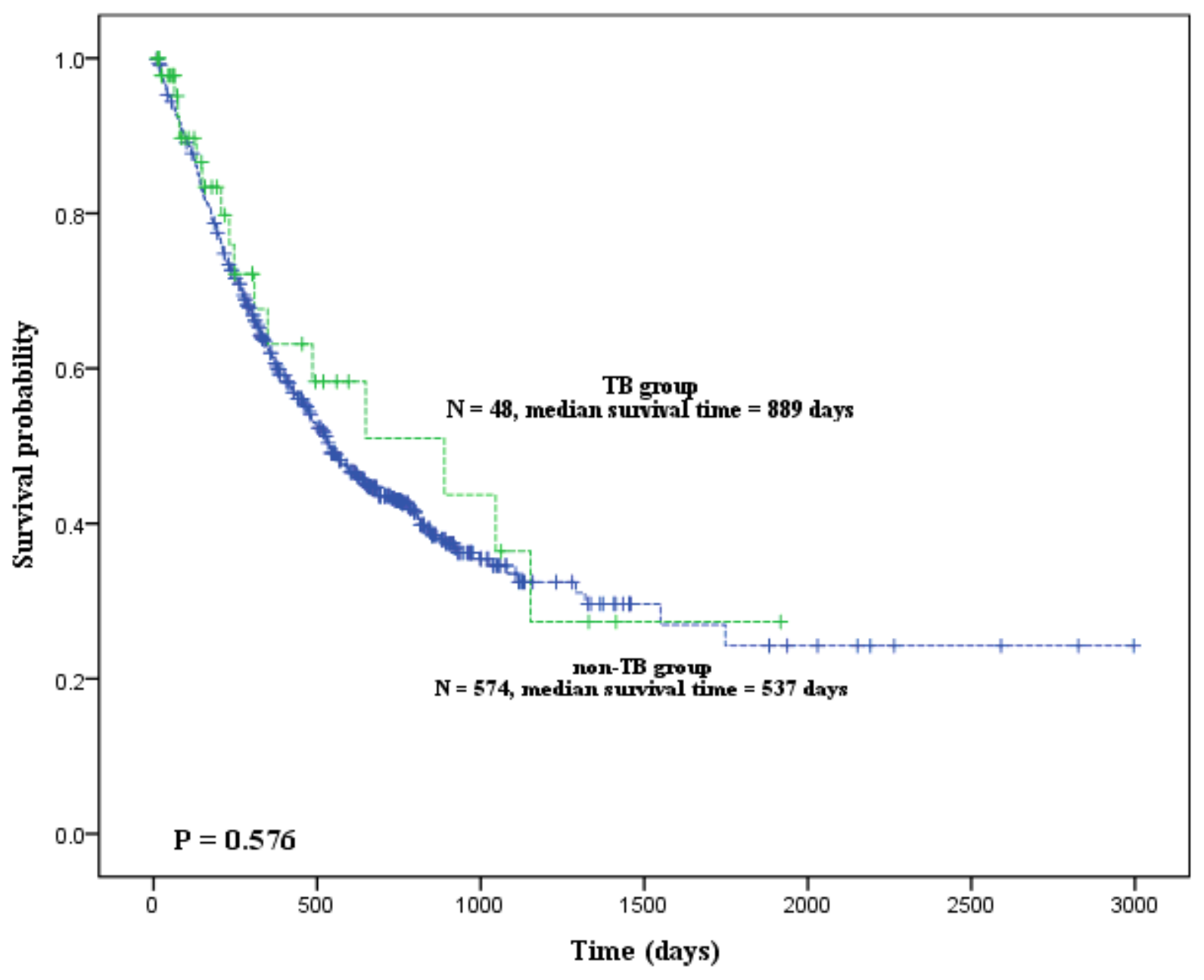

Figure 1

Kaplan-Meier estimate of overall survival of subjects stratified by diagnosis of pulmonary TB. 


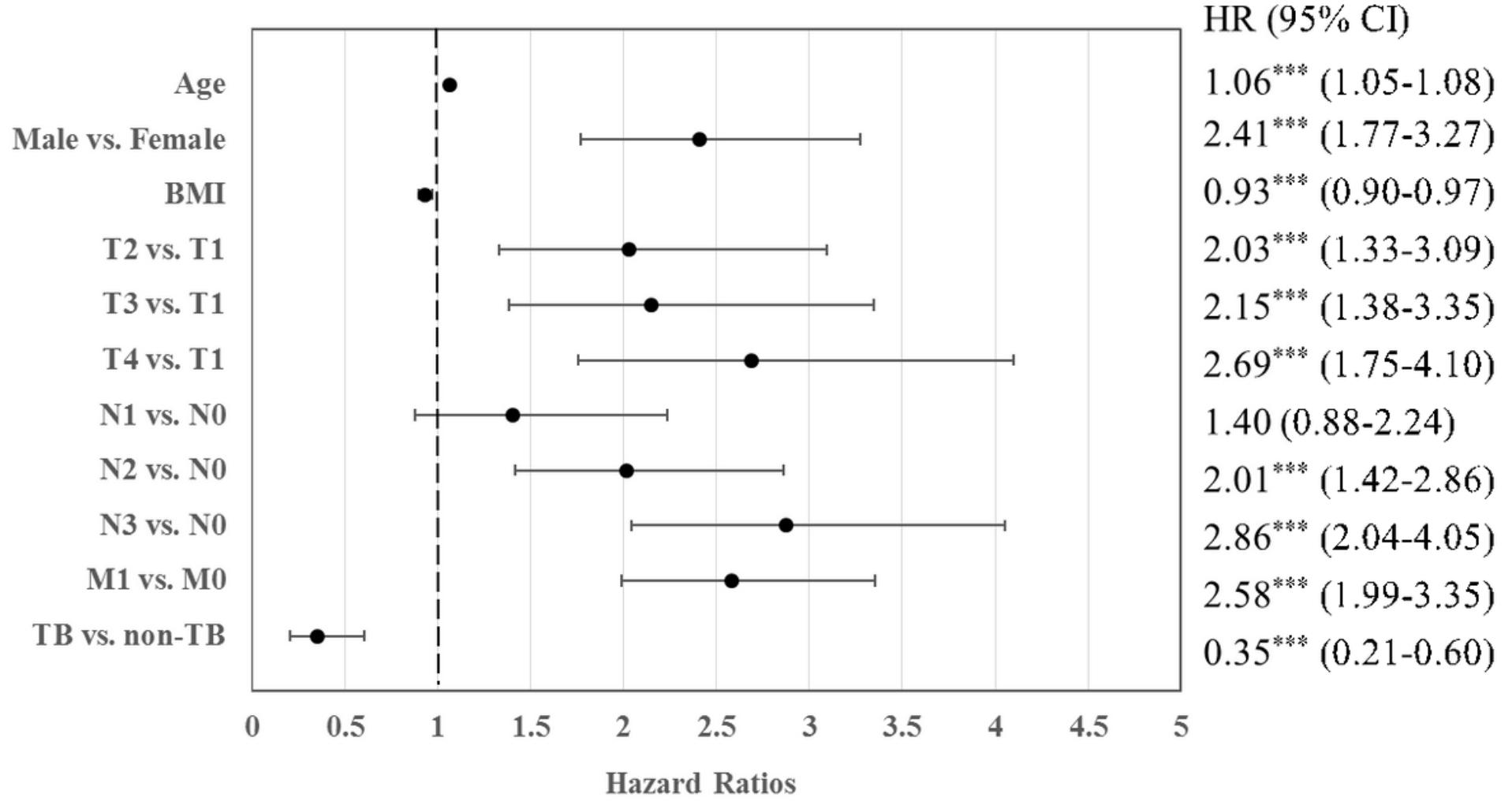

Figure 2

Cox proportional hazards for all-cause mortality 